\title{
Informatics \& Surveillance in Global Health: Informatics Capacity for Zika Outbreak
}

\section{Wycliffe Odongo, Ray Ransom, Olga Henao}

Centers for Disease Control and Prevention, Atlanta, Georgia, United States

\section{Objective}

To assess challenges of establishing surveillance and research study systems and present strategies for rapid deployment in global health for the outbreak response.

\section{Introduction}

In response to the February 2016 Zika virus (ZIKV) outbreak, an inter-agency agreement between the U.S. Centers for Disease Control and Prevention (CDC) and U.S. Agency for International Development (USAID) commissioned further research on the epidemiology, transmission, diagnosis, and birth defects associated with ZIKV. The surveillance and research activities conducted included ecology studies focusing on the transmission dynamics; pregnancy and infant cohort studies to look for birth defects, developmental outcomes and risk factors associated with ZIKV infection; and laboratory studies evaluating the usefulness of multiple Zika diagnostic platforms. These studies were established by either setting up new systems, or leveraging on existing surveillance systems to include ZIKV research specific data elements. Conducted using country-specific protocols, these research systems included key data elements for cross-site analysis. Challenges faced included collection of non-standardized data, differing functional requirements, varying security and confidentiality protocols and limitations of informatics infrastructure. These challenges highlight an opportunity to evaluate and present the informatics-based components necessary to rapidly deploy surveillance and research study activities during a global health emergency situation. We highlight the key challenges and presents strategies for setting up rapid surveillance and research study activities. Additional areas of focus also include system architecture, global partnerships, and workforce development.

\section{Methods}

Information systems used in the ZIKV ecology, pregnancy and diagnostic studies were evaluated in 12 countries in Asia, Africa and the Americas. The research data collection and enrolment for the studies started at different time points (between Feb 2017 and Aug 2017). A baseline survey (structured questionnaire) was administered to the 12 data points of contacts (POCs) in each country to identify existing or selected information systems for use, functional requirements (for data collection, hosting, analytics and integration), existing informatics and infrastructural capacity. Recommendations were made on the selection and configuration of information technology (IT) systems gaps identified in the baseline; with follow up visits to 5 selected sites for intervention implementation as part of CDC's technical assistance. 6 key informant interviews were conducted with subject matter experts on the 6 proprietary(commercial/custom) and 12 semi-structured follow up interviews with data POCs to assess the implementation of the recommendations and interventions. Technical assistance impact was measured by averaging the number of informatics technical assistance requests monthly from the countries over approximately a one year period (Mar 2017- Apr-2018). The Delone and Mclean information system success model was used to measure success . Information quality was scored using completeness, format and timeliness; system quality was scored using availability, adaptability, integration and ease of use; and service quality was measured using reliability and user satisfaction ratings.

\section{Results}

13 (5 open source, 8 proprietary or custom systems) health information systems were identified; 9 exclusively for data collection while 4 had extended functionalities to include extract transform and load (ETL); and, analytics. Selection of these systems was based on awareness and popularity of information technologies in country. Open source systems included REDCap, EpiInfo, DHIS2, Kobo Toolbox, CommCare; while proprietary include University of Virginia's Multi-Schema Information Capture (MuSIC), MS Access, SAMS (CDC's Secure Access Management System) and 3 custom in-house systems. Two (2) Pregnancy study sites (Kenya and Guatemala) used REDCap to enroll and follow up over 1700, and 436 pregnant women respectively while 1 site(Thailand) used a custom web-based Visual Basic system for collecting data on 1000 pregnant women; Ecology studies in 3 sites(Brazil, Colombia and Peru) used smartphones installed with CommCare to collect data on 560 non-human subjects; diagnostic 
studies in 10 sites used existing Acute Febrile Illness (AFI) platforms running custom software, DHIS2, Kobo toolbox, Epi Info, REDCap and Ms Access systems data. Technical assistance (TA) requests were grouped into eight (8) core functional areas with systems design (21.9\%), data transmission and synchronization (18.5\%) and network configuration and diagnostics (13.2\%) identified as key the top 3 areas of TA $(n=820)$. TA requests to CDC ranged between an average of $4($ mean $=4$, s.d $=0.23)$ currently and $11($ mean $=11.25$, s.d $=0.16)$ requests per country per month at the beginning of the pregnancy cohort studies $(n=3)$ in Kenya, Guatemala and Thailand. Diagnostic studies $(\mathrm{n}=10)$ ranged from $26.8(\mathrm{~s} . \mathrm{d}=14.6)$ to $7.5(\mathrm{~s} . \mathrm{d}=4.3)$ while ecology averaged at 1.7 $(\mathrm{N}=3, \mathrm{~s} . \mathrm{d}=1.5)$ requests per country per month. Mean scores of information quality, system quality and service quality were significantly different between sites, as well as between types of information systems $(\mathrm{P}<0.05)$. Total mean scores of information quality, system quality and service quality for were 68.6, 59.8 and 61.4, respectively.

\section{Conclusions}

Robust open source systems exist but their functioalities are not fully exploited. With rapidly changing contexts and outbreak type scenarios, surveillance and research systems must be flexible to rapidly adapt their functional requirements. With appropriate information systems selection guidelines and deliberate informatics technical capacity building we could greatly improve the ability to rapidly deploy systems for outbreak response and global health surveillance and research. Informatics capacity to incorpor ate design thinking and standardization in surveillance system design and implementation could help realize their potential to provide fast and accurate data for action especially in multi-site contexts. Data exchange and security policies across disparate systems in global health,need to be re-aligned with disease surveillance systems' functional requirements.

\section{Acknowledgement}

Ray Ransom, Terrence Lo, Olga Henao and Rama Sima for their help during the data collection and analysis. All the collaborating partners in the Zika research studies.

Disclaimer: The views expressed in this article are those of the authors and do not necessarily reflect the official policy or position of the Centers for Disease Control. 